\title{
Database of Historical Atlases: An Interactive Web Application
}

\author{
Petra Jílková ${ }^{\mathrm{a},} *$, Jiří Cajthaml ${ }^{\mathrm{b}}$ \\ ${ }^{a}$ Czech Technical University in Prague, Faculty of Civil Engineering, Department of Geomatics, Czechia, petra.jilkova@fsv.cvut.cz \\ ${ }^{b}$ Czech Technical University in Prague, Faculty of Civil Engineering, Department of Geomatics, Czechia, jiri.cajthaml@fsv.cvut.cz. \\ * Corresponding author: petra.jilkova@fsv.cvut.cz
}

\begin{abstract}
Within the currently solved project of Czech Historical Atlas, an extensive analytic research of existing historical atlases was conducted with the aim of creating a large database of Czech (Czechoslovak) and foreign historical atlases released after 1950. During the analysis, the team collected information about more than 400 items. Beside the standard bibliographic description (title, author, country of origin, language, availability in the library, etc.), the database contains additional detail information on the content of the atlases, such as a chronological or spatial determination of the maps or applied methods of thematic cartography, that cannot be found in any library catalogue. The database is published as a complementary web application to Czech Historical Atlas web portal. Besides the large amount of collected information, the database of historical atlases in the form of an interactive web application provides a user-friendly visual analytic platform that enables the user to analyse information about historical atlases easily and effectively across fully interactive dashboards. The database can be used not only by academics in the field of historical and atlas cartography but also by broad public interested in this topic. The web application exists in Czech and English version to meet both, Czech and international public.
\end{abstract}

Keywords: historical atlas, cartography, relational database, web application, Czech Historical Atlas

\section{Introduction}

Within the project of Czech Historical Atlas, which is currently solved in the cooperation of the Institute of History of the Czech Academy of Sciences and the Department of Geomatics at Czech Technical University in Prague, the team of historians and cartographers aims to create the web and the printed atlas focused on historical events of the 20th century connected with the Czech lands. The main goal of this project is to present this topic to the public via interactive thematic maps with additional comments and graphics on the web map portal "Czech Historical Atlas". To meet both, the domestic and the international public, the web portal is planned to be available in Czech and English version.

As the concept and the structure of the Czech Historical Atlas follows on from the previous historical atlases, firstly, it was necessary to conduct an extensive analytic research of existing historical atlases of similar focus in order to create a knowledge base for the upcoming atlas. The main output of this research is the large database of more than 400 Czech (Czechoslovak) and foreign historical atlases released after 1950. Although this database was initially meant to serve only as a resource material for the following cartographic work, it was decided to make it available to the broad public. The database was published in the form of an interactive web application enabling simple data filtering and clear visualization of the results.

\section{Database of historical atlases}

\subsection{Methodology concept}

The selection of historical atlases and their characteristics was performed according to predetermined methodology concept given by Bláha, Močičková (2018). The selection was based on two criteria:

1. The researched atlases must be published after 1950 , since they reflect events of the greater part of the 20th century and contain more sophisticated means of map representation in depictions of historical events in compare to earlier issued atlases.

2. The research will cover historical cartographic production in states whose history affected the history of the Czech lands during the 20th century the most, i.e. the Visegrad Group member states (Poland, Slovakia, Hungary) and German-speaking countries bordering Czechia (Germany and Austria); and in states with a significant historical cartographic market such as the United Kingdom, France, Belgium, Switzerland and the United States of America.

\subsection{Data collection}

During the first phase of the research, the atlas works were searched in electronic catalogues of Czech and foreign libraries. As the result, common bibliographic records (e.g. title, authors, country of origin, language, size of atlas, number of pages) of more than 400 items 
were collected. Individual items were also provided with information on the type of atlas (e.g. national, general, military, demographic) that was estimated from the content, and other information available in the catalogue. In addition, a list of libraries where the atlas is physically stored was created for each of the atlases. All collected data from the first phase of the research formed the $1 \mathrm{st}$ level of the database structure. About one quarter of all items (106) account for the atlases of Czech production, the remaining three quarters of the database consist of foreign atlases from other states, mainly from Germany (97), France (65) and the United Kingdom (47). Although the database is not complete, it represents a large and representative sample of the world production of historical atlases during the 2 nd half of the 20th century.

In the second phase of the research (creation of the 2nd level database), 88 out of 409 atlases were selected to be described and analysed in detail, and to be partially digitized. For this purpose, all selected atlases were needed to be physically available for the researchers. Most of the selected atlases, mainly those of Czech production, were found in collections of Czech libraries. Atlases of foreign production that were not located in collections of Czech libraries ( 27 works) were obtained through the international interlibrary loans from selected foreign libraries. The research involved searching for detail information on the content of the atlases (e.g. the amount of maps or the chronological and geographical specification of maps) and analysing applied methods of thematic cartography, the scale series and the map categories according to literature (e.g. Slocum et al., 2008; Voženílek, Kaňok et al., 2011). Subsequently, the basic features (the cover, the front page or the content) and the map excerpts of all selected atlases were digitized. At the end, the researchers searched for images of covers of the remaining atlases on the web to complete the database.

\section{Web application}

Although it was not initially intended to open the output of the research to the public, the authors concluded that the database could be useful for further research activities and decided to publish the database as a complementary web application to Czech Historical Atlas web portal (http://cha.fsv.cvut.cz/web/). The main aim was to provide not just a simple database of tabular data but an interactive database application that would dynamically respond to user interaction and would demand a low cognitive effort of the user. For this purpose, a dynamic web application enabling simple data filtering and clear visualization of the results was designed by one of the CTU students within her diploma thesis (Vévodová 2017).

\subsection{Database structure}

Firstly, it was necessary to design the structure of the relational database to remove the data redundancy as the collected data were originally stored in one large table. Thus the original table was split into 11 tables. The main table "Publications" where all attributes about the atlases are stored was linked to 10 smaller tables storing the attributes about the chosen subjects:

- authors ("Authors")

- publishing country ("States")

- type of atlas (“AtlasTypes")

- geographical determination of maps ("Regions")

- used cartographic method ("Methods")

- publisher ("Publishers")

- main language of the atlas ("Languages")

- name of the library ("Libraries")

- colours of the map ("Colors")

- map categories ("Categories")

Each of these tables is related to the main table via the ID attribute. Figure 1 shows the structure of the tables and the relations. All tables are stored in Google Sheets according to the predefined structure of the database to be freely available for the users of the web application. Each table is represented by a single sheet. In order to link the downloaded and digitized images of the atlases to the database, two new columns ("images" and "content") containing the path of the image file were added to the main table ("Publications"). During the creation of the web application, the structure of the database was further edited to ensure the correct functionality of the application.

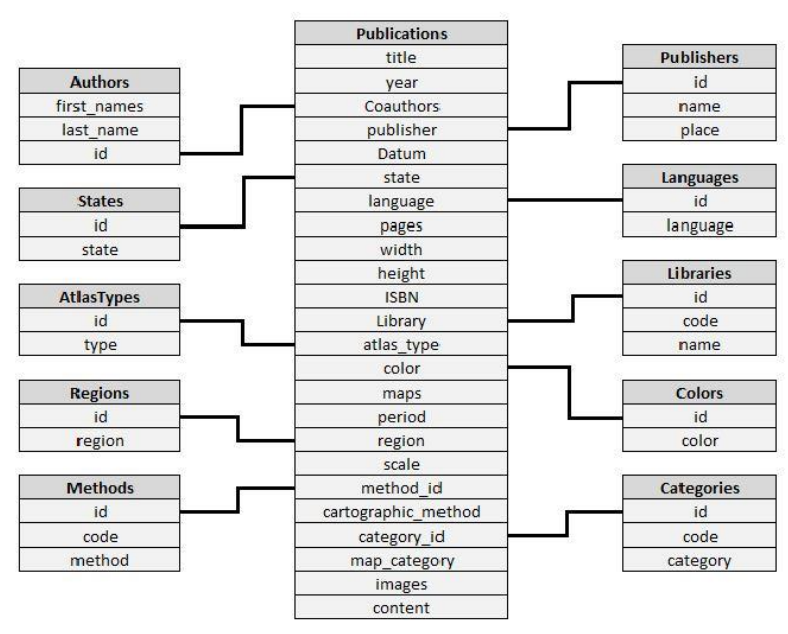

Figure 1. Relationships between tables in the database (Vévodová 2017)

\subsection{Technical solution}

Among many available data publishing solutions, the author selected an existing database application for publications created with Keshif - a JavaScript library that provides an automated and systematic web-based interface for data exploration and that can be integrated or used as a web service enabling tabular data exploration. The source code of the sample application was then configured and customized using basic web programming (JavaScript, HTML, CSS) and other JavaScript libraries under open-source license such as the D3 library, the jQuery library, the JSAPI library and the Moment library. The mentioned JavaScript libraries were chosen as the 
most convenient to ensure the desired functionality of the web application for following reasons:

- D3.js - enables to transform arbitrary data tables into visual graphic such as interactive charts, diagrams or animations, and can be easily used with HTML and CSS standards

- jQuery.js - simplifies the manipulation of HTML, animations or AJAX, that enables to change the content of the web application dynamically with no need to reload the page

- Moment.js - enables to manipulate and display dates and time in JavaScript; therefore, it was used to filter the release dates of the atlases

In addition, most of these libraries supports the most commonly used web browsers (Google Chrome, Mozilla Firefox, Safari, Microsoft Edge or Internet Explorer).

\subsection{User interface}

The web application consists of 10 interactive dashboards as shown in Figure 2. The main dashboard panel is situated in the middle and contains a list of all 409 historical atlases sorted by the release date in descending order. Each of records can be expand to show the standard bibliographic description (title, author, country of origin, language, availability in the library, etc.) and the focus category of the individual atlas including the preview of the cover. Some of the records contain additional information such as the chronological and spatial determination of maps, the applied method of thematic cartography, or the scale and categories of maps. Links to digitized images of the front page, the content, or the map excerpts are also included for some records analysed in the second phase of the research (see section 2.2 above). User can easily adjust the size of the main dashboard panel by dragging the dotted line along its left side. Through the main dashboard panel, the user can also open the original dataset in Google Sheets.
The user can search an individual atlas by its name or any keyword via the search panel, or select the atlas by predefined attributes in dashboard panels that are situated along the main dashboard panel. The application allows the user to filter the atlases by the author, publisher, publishing country, language, library, type of atlas, cartographic method or map category. Filters are applied directly in the dashboards by clicking on the desired category. Another category can be added to filter in the same way (AND condition) or by clicking on plus (OR condition) or minus (NOT condition) signs that are displayed on mouse-over. All applied filters are displayed at the top bar of the main dashboard and can be easily removed from there. In each dashboard panel coloured bar charts or histograms show the distribution of data in the category. The absolute number of records per each category is shown as well. The content of the dashboards is fully synchronised and responsive - the categories are dynamically ordered after filtering to reveal the most common categories first. The web application also allows so called "rapid highlighting" that enables to instantly select or filter the atlases only by a mouse-over, dynamically highlight associated information across all dashboards and update the content of the dashboard according to the selected category. This function enables the user to reveal information about the atlas without the need to expand its record in the main dashboard.

\subsection{Web browsers}

Before publishing, the performance of the web application was tested in the six most commonly used desktop web browsers in Czechia and worldwide according to StatCounter (2019). The usage shares of the six most commonly used browsers are summarized in Table 1. Although there exist special tools for testing the performance of web applications in web browsers, they are more convenient for testing the large-scale projects. Thus the performance of the web application was simply tested by launching it in the particular web browser.

\section{Database of Historical Atlases Since 2nd Half of the 20th Century}

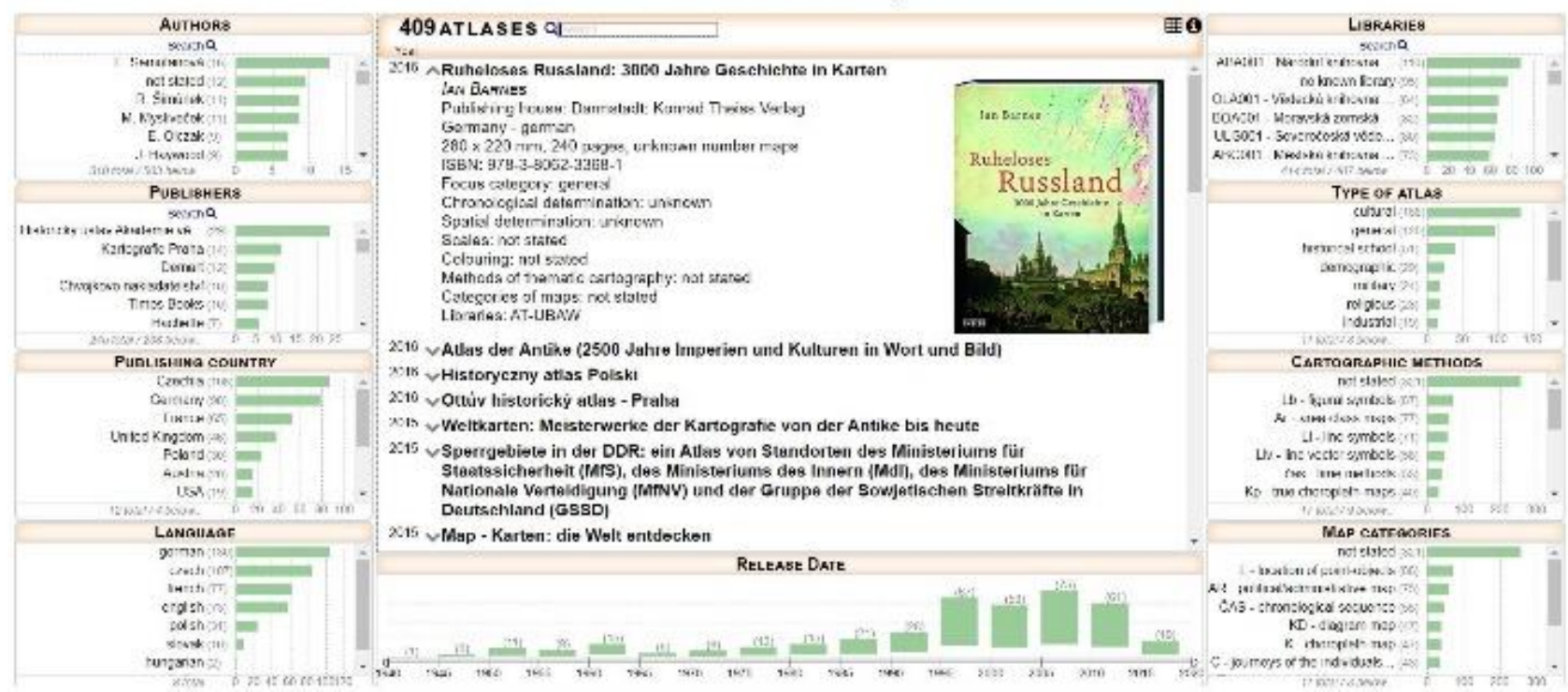

Figure 2. The basic layout of the web application in Firefox 
The web application performs the best in Google Chrome and Opera. Both web browsers load the web application very fast and display the results of searching and filtering process in the shortest time. Although in Mozilla Firefox, Internet Explorer and Microsoft Edge the web application responds to user activity more slowly, these web browsers are still suitable for running the application. The least convenient (and thus not recommended) web browser for running the web application is Safari. In Safari, the web application runs very slowly or is not working at all. In other web browsers except Mozilla Firefox, the layout of the web application may slightly differ from the one shown in Figure 1.

\begin{tabular}{|l|r|r|}
\hline web browser & Czechia & worldwide \\
\hline Google Chrome & $60,95 \%$ & $69,51 \%$ \\
Mozilla Firefox & $16,44 \%$ & $9,57 \%$ \\
Internet Explorer & $6,82 \%$ & $5,48 \%$ \\
Microsoft Edge & $5,14 \%$ & $4,56 \%$ \\
Opera & $5,13 \%$ & $2,43 \%$ \\
Safari & $4,62 \%$ & $6,44 \%$ \\
other & $0,90 \%$ & $2,01 \%$ \\
\hline
\end{tabular}

Table 1. Usage share of browsers in Czechia and worldwide

\section{Conclusion}

The final structure of the database represents a complex and rich source of data about historical atlases. The user can search in the database not only for the standard bibliographic data (title, author, country of origin, language, availability in the library, etc.) but also for additional information on selected atlases that cannot be found in any standard library catalogue, such as the chronological and geographical determination of maps or applied methods of thematic cartography. Besides the large amount of information collected in one place, the database of historical atlases in the form of an interactive web application also meets the demands of the modern user. It provides an attractive visual analytic platform that enables the user to analyse information about historical atlases easily and effectively across fully interactive dashboards without the need to browse catalogues of individual libraries. The database can be used not only by academics in the field of historical and atlas cartography but also by broad public interested in this topic. Since the web application exists in Czech and English version, it can be used by both, Czech and international public. The web application is available at http://maps.fsv.cvut.cz/atlases/. It is recommended to run the application in Google Chrome, Opera, Mozilla Firefox, Internet Explorer or Microsoft Edge.

\section{Acknowledgements}

This work was supported by the Czech Ministry of Culture from the NAKI - DG16P02H010 project "Czech Historical Atlas".

\section{References}

Bláha, J. D. and Močičková, J. (2018). The ResearchAnalytic Part of Preparation of a Cartographic Work: A Case Study of an Analysis of Historical Atlases as the Basis for Creating the Czech Historical Atlas. AUC Geographica, 53(1), 58-69, ISSN 2336-1980.

Slocum, T. A., McMaster, R. B., Kessler, F. C. and Howard, H. H. (2008). Thematic Cartography and Geographic Visualization. Upper Saddle River, Pearson/Prentice Hall.

StatCounter (2019, March 30). Browser Market Share. Retrieved from: http://gs.statcounter.com/

Vévodová, P. (2017): Databáze historických atlasů. Diploma thesis. Czech Technical University in Prague.

Voženílek, V., Kaňok, J. et al. (2011): Metody tematické kartografie: Vizualizace prostorových jevů. Olomouc, Vydavatelství Univerzity Palackého. 